\title{
Gradings of periodontitis in relation with systemic disease
}

\author{
Abdulhameed G. Albeshr ${ }^{1 *}$, Yazeed A. Alhabdan', Raef Mohamad Aljasem², \\ Maha Assad Munshi ${ }^{2}$, Ahmed Mohammadhassan Bagasi ${ }^{3}$, Mohammed Yaseer Al Khars ${ }^{4}$, \\ Naif Mayouf Alrasheedi ${ }^{5}$, Mohammed Bandar Alodiani ${ }^{6}$, Mohammed Abdulrahman Alnefaie ${ }^{6}$, \\ Wesam Yousef Alibrahim ${ }^{7}$, Reem Mohammed Alharbi ${ }^{8}$, Hadeel Fahad Aljuaid9
}

\author{
${ }^{1}$ King Abdullah Medical City for National Guard, Riyadh, Saudi Arabia \\ ${ }^{2}$ General Dentist, Ministry of Health, Jeddah, Saudi Arabia \\ ${ }^{3}$ College of Dentistry, Umm Al-Qura University, Mecca, Saudi Arabia \\ ${ }^{4}$ College of Dentistry, King Fasial University, Al Ahsa, Saudi Arabia \\ ${ }^{5}$ General Dentist, Ministry of Health, Uyun Aljaw, Saudi Arabia \\ ${ }^{6}$ College of Dentistry, Majmaah University, Majmaah, Saudi Arabia \\ ${ }^{7}$ General Dentist, Dental Medical Complex in West of Riyadh, Riyadh, Saudi Arabia \\ ${ }^{8}$ General Dentist, Unidents Clinic, Jeddah, Saudi Arabia \\ ${ }^{9}$ College of Dentistry, Batterjee Medical College, Jeddah, Saudi Arabia
}

Received: 15 June 2021

Accepted: 30 June 2021

\section{*Correspondence:}

Dr. Abdulhameed G. Albeshr,

E-mail: agaab2005@hotmail.com

Copyright: (C) the author(s), publisher and licensee Medip Academy. This is an open-access article distributed under the terms of the Creative Commons Attribution Non-Commercial License, which permits unrestricted non-commercial use, distribution, and reproduction in any medium, provided the original work is properly cited.

\begin{abstract}
With the increasing prevalence rates of periodontitis cases, it has a great impact on human health, especially elderly patients. It constitutes a major health problem that can cause significant burdens. Infections are the main pathology that have been previously reported among studies in the literature because it has a strong correlation. There are common mechanisms that can cause systemic diseases: induction of systemic inflammation, inflammatory markers, the release of toxins, and translocation into other organs through the bloodstream. Proving the correlation between the potential presence of the involved pathogen and systemic diseases might be a significant key factor in predicting these systemic events. In addition, it will assist to have proper management in early intervention. The aim of the study was to provide more insight into the effect of correlation between periodontitis and other systemic diseases according to studies in the literature. Multiple organisms that can cause periodontitis have been significantly correlated with the development of many systemic diseases, such as atherosclerosis, coronary artery disease (CAD), pneumonia, respiratory tract infections, oral and colorectal cancers, diabetes and Alzheimer's disease. Moreover, Porphyromonas gingivalis has been commonly reported with the variously discussed systemic diseases which should draw more attention towards the proper management and prevent this organism and other microbes within the literature to enhance the outcomes.
\end{abstract}

Keywords: Periodontitis, Dentistry, Complications, Systemic, Inflammation

\section{INTRODUCTION}

It has been previously estimated that periodontitis and related diseases are common with inflammatory disorders and is found to affect 3.9 billion patients globally in 2010 . Estimates also showed that the prevalence of moderate-to- severe periodontitis is $11 \%$ while mild periodontitis accounts for $35 \% .{ }^{1}$ With the increasing prevalence rates of periodontitis cases, it constitutes a major health problem that can cause significant burdens. ${ }^{2}$ Previous research by the United States Centers for Disease Control and Prevention has suggested that periodontitis is considered a pandemic disease that can cause serious morbidities and 
impact the affected patients' quality of life. ${ }^{2}$ Previous research by the United States Centers for Disease Control and Prevention has suggested that periodontitis is considered a pandemic disease that can cause serious morbidities and impact the affected patients' quality of life. $^{3}$

Nevertheless, non-dentistry-related investigations have been extensively found in the literature regarding periodontitis for the impact that the disease might have on the initiation and effect on other systemic diseases. Such investigations include cardiovascular diseases, respiratory tract infections, colorectal cancer, type 2 diabetes mellitus, and other oral diseases. ${ }^{4-6}$ Proving the correlation between the potential cause and the occurrence of systemic diseases might be a significant key factor in predicting these systemic events. In addition, it will assist to have proper management in early intervention. Accordingly, this present literature review aims to provide more insight into the effect of correlation between periodontitis and other systemic diseases.

\section{METHODS}

A systematic search was conducted to identify relevant studies in the following databases: PubMed, Medline, Web of Science, Embase, Google Scholar, and Scopus. The following search terms were used ('periodontitis' or 'aggressive periodontitis') and ('grading') and ('systemic' or 'systemic disease') and ('relation' or 'association'). The reference lists were manually searched to identify additional relevant studies meeting inclusion criteria. We included any study that reports post grading of periodnotitis and its relation to systemic diseases. No restrictions were applied.

\section{DISCUSSION}

\section{Cardiovascular diseases}

Previous epidemiological investigations have reported that cardiovascular diseases might be associated with periodontitis due to major controversies regarding the potential effect that infections might have on the development and prognosis of cardiovascular diseases. A previous meta-analysis investigated the correlation between having periodontal diseases and developing coronary artery disease (CAD) among 86,092 patients that were recruited by five cohort investigations and found that the risk of developing CAD increased by 1.12 folds among patients with periodontal diseases. ${ }^{7}$ Moreover, a previous large case-control study also reported that the risk was even greater, attributing to a 2.22 increased folds for patients with periodontal diseases. ${ }^{7}$ Another observational investigation of 1,163 male patients previously reported that CAD was significantly associated with serum antibodies against A. actinomycetemcomitans and Prophyromonas gingivalis and edentulousness. ${ }^{8}$ In the same context, another study that performed endarterectomy showed that the DNA pathogenic bacterial species were found among 42 obtained atheromatous plaques. ${ }^{9}$ The most commonly reported bacterial species by the authors were Porphyromonas gingivalis. Other causes included are A. actinomycetemcomitans, Eikenella corrodens, T. forsythia, Campylobacter rectus, and Fusobacterium nucleatum. ${ }^{9}$ In addition, previous investigations have also reported that these oral bacterial strains were significantly observed among patients with atherosclerosis, indicating a strong association. ${ }^{10,11}$ Moreover, previous animal studies have demonstrated that atherosclerosis and alveolar bone loss were significantly observed among mice with hyperlipidemia that was infected with Porphyromonas gingivalis and treponema denticola. Furthermore, a possible correlation was indicated when they noticed the significant immune response after induction of the bacterial infection by observing the DNA and bacterial strains of the infecting bacteria in the oral epithelium, in addition to other systemic organs, including the Aorta. ${ }^{12,13} \mathrm{~A}$ previous investigation also reported that chronic inflammation within the vascular tissue was previously reported with Porphyromonas gingivalis through induction of Toll-like receptor (TLR)-4 and activation of innate immunity. ${ }^{14} \mathrm{~A}$ previous in vivo investigation also reported that Porphyromonas gingivalis was associated with thrombus formation. Therefore, it was suggested that the pathogen is capable of activating platelet aggregation in the body through the vesicles which are located on their outer membranes. ${ }^{15}$ However, another investigation has reported that other oral pathogenic bacteria that caused periodontitis were not able to cause platelet aggregation, which is suggestive that only Porphyromonas gingivalis is capable of causing this manifestation. ${ }^{16}$

\section{Pneumonia and respiratory tract infections}

It is now well-known that oral bacterial infections can contribute in inducing pneumonia and respiratory tract infections, which are the main causes of severe morbidity and mortality in many patients across the globe, especially the elderly and immunocompromised individuals. Previous investigations in the literature reported the involvement of many oral organisms in the pathology and developing infections that affect the respiratory tract. These include A. actinomycetemcomitans, Capnocytophaga spp, Actinomyces israelii, Chlamydia pneumoniae, E. corrodens, Fusobacterium necrophorum, F. nucleatum, P. gingivalis, Streptococcus constellatus and P. intermedia. ${ }^{17-19}$ A previous investigation in the intensive care unit showed that patients with severe respiratory tract infections who underwent bronchoalveolar lavage the same bacterial strains that were detected in their oral plaques. ${ }^{20} \mathrm{~A}$ previous investigation also reported that having periodontitis was significantly associated with a three-fold increase in catching nosocomial infections than others do not have it. ${ }^{21}$ A previous animal study also reported that inducing $\mathrm{P}$. gingivalis infections in the included sample was associated with a significant inflammatory response within the lungs and estimated proinflammatory cytokines production and cellular 
aggregation. ${ }^{17}$ Another observational study that recruited 40 patients reported that orotracheal intubation showed that $\mathrm{P}$. gingivalis, A. actinomycetemcomitans, and $\mathrm{T}$. forsythia were extensively have seen in the obtained samples suggesting that these bacteria play a significant role in respiratory tract infections, even within toothless patients as the authors reported. ${ }^{22}$ Besides, it has also been previously reported that $\mathrm{F}$. necrophorum and F. nucleatum were significantly associated with induction of a series of conditions, starting with pharyngitis which can lead to Lemierre's syndrome as well as respiratory tract infection. ${ }^{18,19}$ A previous cross-sectional study that was conducted among school students reported that $\mathrm{F}$. necrophorum was detectable in patients that suffered from acute sore throats even more than group A $\beta$-hemolytic streptococcus, as it was detectable in $20.5 \%$ and $9.4 \%$ compared to $10.3 \%$ and $1.1 \%$ for streptococcus in symptomatic and asymptomatic patients, respectively. ${ }^{23}$ Therefore, it could be suggested that fusobacterium infections are common among patients with respiratory tract infections that should be considered when planning for adequate management. C. pneumoniae has also been previously reported as a common cause of chronic obstructive pulmonary diseases and asthma. ${ }^{24}$ Studies suggest that the pathogen can be frequently detected within the oral cavity and can easily translocate to the lower respiratory epithelium, in addition to its capability of moving and infecting other systemic organs such as the heart, spleen and aorta. According to a previous animal study, it showed that this pathogenesis was achievable through the monocytes in the circulation. ${ }^{25-27}$ A previous investigation also reported that the pathogen is capable of inducing atherosclerosis, which might also add to the previously discussed association between oral pathogens and the development of atherosclerosis and other systemic diseases. ${ }^{28}$

\section{Colorectal and oral cancers}

Studies suggest that colorectal cancer is significantly associated with being infected with $\mathrm{F}$. nucleatum and $\mathrm{C}$. difficile. $^{29}$ Additionally, previous studies have also demonstrated the potentiality of other organisms as Leptotrichia, Fusobacterium and Campylobacter species. ${ }^{30}$ Kostic et al studied the correlation between F. nucleatum and incidence of colorectal cancer in animals and reported that F. nucleatum was significantly associated with the progression of colorectal carcinoma through enhancement of the actions of the myeloid infiltrating cells by multiplying them. ${ }^{31}$ The same study reported that the same microbe could alter the beneficial effects of the bowel microbiota, increase the expression of harmful cytokines and activate tumorigenesis-related pathways that can significantly lead to the development of colorectal carcinoma. Therefore, it could be suggested that F. nucleatum can significantly induce colorectal adenomacarcinoma in humans. ${ }^{32}$ Infections can pose a significant risk factor for the development of cancer. Helicobacter pylori was previously reported to be a significant risk factor as it was associated with the development of gastric carcinoma. ${ }^{33,34}$ Concerning periodontitis, a previous metaanalysis that analyzed the outcomes of 3,183 patients with periodontal diseases showed that infections causing periodontitis were significantly associated with the development of oral cancers. ${ }^{35}$ Additionally, other investigations also reported that periodontal infections were significantly associated with the development of malignancy in the lung, head of pancreas and neck of pancreas. ${ }^{34}$ A previous Taiwanese comparative investigation of one million participants reported that patients in the gingivitis group had a lower risk of developing oral cancer than patients within the periodontitis group, who had a higher risk. ${ }^{36}$ Previous studies have also demonstrated the frequent observation of the $P$. gingivalis pathogen within the oral mucosa that were detected in patients with esophageal squamous cell carcinoma, and squamous cell carcinoma. However, they were not found within the normal mucosa. ${ }^{37,38}$ A previous animal study also reported the same phenomenon by showing the potential pathogenic roles that these organisms have in the development of oral cancer. They showed that a process of carcinogenesis which is medicated by the patient's innate immunity can be significantly observed with F. nucleatum and P. gingivalis, which have been found to directly interact with the underlying epithelium. ${ }^{39}$ A previous investigation also reported that $\mathrm{P}$. gingivalis also plays an important role by activating the matrix metalloproteinase 9 (pro-MMP9) which increases the invasion and metastasis of the oral squamous cell carcinoma; however, this mechanism was not noticed with F. nucleatum. ${ }^{37}$ Similarly, Gallimidi et al studied the same organism and found that it was capable of inducing the aggressiveness of the same carcinoma through enhancing the mesenchymal transition activities of the carcinoma. ${ }^{40}$ Therefore, these two pathogens should be seriously considered as biomarkers for developing oral disease and malignancy and should be used as early preventive measures against the future development of such disorders.

\section{Diabetes mellitus and Alzheimer's disease}

Regarding diabetes, chronic periodontitis can significantly negatively impact the metabolism of blood sugar, leading to disturbances in insulin secretion and the development of diabetes. ${ }^{41}$ This can be translated to similar idea that patients with viral infections can significantly suffer from insulin resistance. ${ }^{42}$ According to Fernandez et al, C. pneumoniae and enteroviruses were significantly associated with middle-grade inflammatory responses that were associated with insulin resistance. ${ }^{43} \mathrm{~A}$ meta-analysis study in 2010 concluded that management of periodontal disorders was significantly associated with improved glycemic control in type 2 diabetes mellitus. ${ }^{44}$ As a result, we can propose that periodontitis and poor glycemic control in diabetes are connected and may occur concurrently. On the other hand, it has been found that diabetes can also be complicated by microvascular complications, including periodontitis and other oral infections. A previous study reported that periodontitis can 
be significantly anticipated by a three-fold increase among patients with diabetes than the general population. ${ }^{45}$ Another observational study by Xavier et al in 2009 reported a periodontitis prevalence rate of $6 \%$ among their diabetic population while type 1 diabetes for more than five years was associated with an increased risk of multiple periodontal lesions. ${ }^{46}$

Regarding Alzheimer's disease, evidence also shows that periodontitis might be linked to Alzheimer's and vice versa. A previous investigation reported that chronic periodontitis and poor oral health were frequently observed among patients with increased cognitive decline. ${ }^{47}$ Increased expression of chemokines and cytokines and complement activation are mainly stated to cause Alzheimer's disease while immune-mediated mechanisms have not been previously validated. ${ }^{48}$ Therefore, inflammation and neurodegeneration through $\beta$-amyloid plaques are significantly associated with the development of Alzheimer's disease, as it connected to patients with periodontitis. ${ }^{49}$ Besides, T. denticola, C. pneumoniae, and $\mathrm{P}$. gingivalis, in addition to having a pro-inflammatory mechanism in activating the development of Alzheimer's disease, have also been previously found within the parenchyma of the brain in post-mortem analysis of cases that suffered from Alzheimer's disease. ${ }^{50}$ Additionally, antibodies against $\mathrm{P}$. gingivalis, A. actinomycetemcomitans, T. forsythia, and P. intermedium were significantly prevalent in patients with Alzheimer's disease. ${ }^{50}$

\section{CONCLUSION}

Periodontal infections are associated with the development of other systemic diseases. Multiple organisms that can cause periodontitis have been significantly correlated with the development of many systemic diseases like atherosclerosis and CAD, pneumonia and respiratory tract infections, oral and colorectal cancers, diabetes, and Alzheimer's disease. Early prevention and proper management of the disease can be key elements in the intervention against the development of such diseases and to enhance the affected patients' outcomes.

Funding: No funding sources

Conflict of interest: None declared

Ethical approval: Not required

\section{REFERENCES}

1. Richards D. Oral diseases affect some 3.9 billion people. Evid Based Dent. 2013;14(2):35.

2. CDC researchers find close to half of American adults have periodontitis. J Can Dent Assoc. 2012;78:136.

3. Socransky SS, Haffajee AD, Cugini MA, Smith C, Kent RL Jr. Microbial complexes in subgingival plaque. J Clin Periodontol. 1998;25(2):134-44.

4. Whitmore SE, Lamont RJ. Oral bacteria and cancer. PLoS Pathog. 2014;10(3):1003933.
5. Yeo BK, Lim LP, Paquette DW, Williams RC. Periodontal disease- the emergence of a risk for systemic conditions: pre-term low birth weight. Ann Acad Med Singap. 2005;34(1):111-6.

6. Kim J, Amar S. Periodontal disease and systemic conditions: a bidirectional relationship. Odontology. 2006;94(1):10-21.

7. Bahekar AA, Singh S, Saha S, Molnar J, Arora R. The prevalence and incidence of coronary heart disease is significantly increased in periodontitis: a metaanalysis. Am Heart J. 2007;154(5):830-7.

8. Pussinen PJ, Jousilahti P, Alfthan G, Palosuo T, Asikainen S, Salomaa V. Antibodies to periodontal pathogens are associated with coronary heart disease. Arterioscler Thromb Vasc Biol. 2003;23(7):1250-4.

9. Figuero E, Sanchez BM, Cuesta FS, Tejerina JM, Castro JA, Gutierrez JM, et al. Detection of periodontal bacteria in atheromatous plaque by nested polymerase chain reaction. J Periodontol. 2011;82(10):1469-77.

10. Haraszthy VI, Zambon JJ, Trevisan M, Zeid M, Genco RJ. Identification of periodontal pathogens in atheromatous plaques. J Periodontol. 2000;71(10):1554-60.

11. Nakano K, Inaba H, Nomura R, Nemoto H, Takeda $\mathrm{M}$, Yoshioka $\mathrm{H}$, et al. Detection of cariogenic Streptococcus mutans in extirpated heart valve and atheromatous plaque specimens. J Clin Microbiol. 2006;44(9):3313-7.

12. Chukkapalli SS, Rivera MF, Velsko IM, Lee JY, Chen H, Zheng D, et al. Invasion of oral and aortic tissues by oral spirochete Treponema denticola in ApoE(-/-) mice causally links periodontal disease and atherosclerosis. Infect Immun. 2014;82(5):1959-67.

13. Velsko IM, Chukkapalli SS, Rivera MF, Lee JY, Chen H, Zheng D, et al. Active invasion of oral and aortic tissues by Porphyromonas gingivalis in mice causally links periodontitis and atherosclerosis. PLoS One. 2014;9(5):97811.

14. Slocum C, Coats SR, Hua N, Kramer C, Papadopoulos G, Weinberg EO, et al. Distinct lipid a moieties contribute to pathogen-induced site-specific vascular inflammation. PLoS Pathog. 2014;10(7):1004215.

15. Herzberg MC, Meyer MW. Effects of oral flora on platelets: possible consequences in cardiovascular disease. J Periodontol. 1996;67(10):1138-42.

16. Sharma A, Novak EK, Sojar HT, Swank RT, Kuramitsu HK, Genco RJ. Porphyromonas gingivalis platelet aggregation activity: outer membrane vesicles are potent activators of murine platelets. Oral Microbiol Immunol. 2000;15(6):393-6.

17. Hajishengallis G, Wang M, Bagby GJ, Nelson S. Importance of TLR2 in early innate immune response to acute pulmonary infection with Porphyromonas gingivalis in mice. J Immunol. 2008;181(6):4141-9.

18. Sonti R, Fleury C. Fusobacterium necrophorum presenting as isolated lung nodules. Respir Med Case Rep. 2015;15:80-2. 
19. Williams MD, Kerber CA, Tergin HF. Unusual presentation of Lemierre's syndrome due to Fusobacterium nucleatum. J Clin Microbiol. 2003;41(7):3445-8.

20. Heo SM, Sung RS, Scannapieco FA, Haase EM. Genetic relationships between Candida albicans strains isolated from dental plaque, trachea, and bronchoalveolar lavage fluid from mechanically ventilated intensive care unit patients. J Oral Microbiol. 2011;3.

21. Filho IS, Oliveira TF, Cruz SS, Passos SS, Trindade SC, Oliveira MT, et al. Influence of periodontitis in the development of nosocomial pneumonia: a case control study. J Periodontol. 2014;85(5):82-90.

22. Porto AN, Borges AH, Rocatto G, Matos FZ, Borba AM, Pedro FL, et al. Periodontal and Microbiological Profile of Intensive Care Unit Inpatients. J Contemp Dent Pract. 2016;17(10):807-14.

23. Centor RM, Atkinson TP, Ratliff AE, Xiao L, Crabb DM, Estrada CA, et al. The clinical presentation of Fusobacterium-positive and streptococcal-positive pharyngitis in a university health clinic: a crosssectional study. Ann Intern Med. 2015;162(4):241-7.

24. Roulis E, Polkinghorne A, Timms P. Chlamydia pneumoniae: modern insights into an ancient pathogen. Trends Microbiol. 2013;21(3):120-8.

25. Mantyla P, Stenman M, Paldanius M, Saikku P, Sorsa T, Meurman JH. Chlamydia pneumoniae together with collagenase-2 (MMP-8) in periodontal lesions. Oral Dis. 2004;10(1):32-5.

26. Filardo S, Pietro M, Schiavoni G, Minniti G, Ortolani E, Romano S, et al. Chlamydia pneumoniae Clinical Isolate from Gingival Crevicular Fluid: A Potential Atherogenic Strain. Front Cell Infect Microbiol. 2015;5:86.

27. Yamaguchi H, Oshio I, Osaki T, Kurata S, Yamamoto Y, Kamiya S. Development of diabetes in non-obese diabetic mice promotes Chlamydia pneumoniae dissemination from lung to peripheral blood. Int J Exp Pathol. 2006;87(2):121-9.

28. Campbell LA, Rosenfeld ME. Infection and Atherosclerosis Development. Arch Med Res. 2015;46(5):339-50.

29. Fukugaiti MH, Ignacio A, Fernandes MR, Ribeiro JU, Nakano V, Avila CMJ. High occurrence of Fusobacterium nucleatum and Clostridium difficile in the intestinal microbiota of colorectal carcinoma patients. Braz J Microbiol. 2015;46(4):1135-40.

30. Warren RL, Freeman DJ, Pleasance S, Watson P, Moore RA, Cochrane K, Allen VE, et al. Cooccurrence of anaerobic bacteria in colorectal carcinomas. Microbiome. 2013;1(1):16.

31. Kostic AD, Chun E, Robertson L, Glickman JN, Gallini CA, Michaud M, et al. Fusobacterium nucleatum potentiates intestinal tumorigenesis and modulates the tumor-immune microenvironment. Cell Host Microbe. 2013;14(2):207-15.

32. Yu YN, Yu TC, Zhao HJ, Sun TT, Chen HM, Chen $\mathrm{HY}$, et al. Berberine may rescue Fusobacterium nucleatum-induced colorectal tumorigenesis by modulating the tumor microenvironment. Oncotarget. 2015;6(31):32013-26.

33. Thieu H, Bach DB, Nam NH, Reda A, Duc NT, Alshareef A, Nguyen JG, et al. Antibiotic resistance of Helicobacter pylori infection in a children's hospital in Vietnam: prevalence and associated factors. Minerva Med. 2020;111(5):498-501.

34. Michaud DS, Fu Z, Shi J, Chung M. Periodontal Disease, Tooth Loss, and Cancer Risk. Epidemiol Rev. 2017;39(1):49-58.

35. Yao QW, Zhou DS, Peng HJ, Ji P, Liu DS. Association of periodontal disease with oral cancer: a meta-analysis. Tumour Biol. 2014;35(7):7073-7.

36. Wen BW, Tsai CS, Lin CL, Chang YJ, Lee CF, Hsu $\mathrm{CH}$, et al. Cancer risk among gingivitis and periodontitis patients: a nationwide cohort study. QJM. 2014;107(4):283-90.

37. Inaba H, Sugita H, Kuboniwa M, Iwai S, Hamada M, Noda T, et al. Porphyromonas gingivalis promotes invasion of oral squamous cell carcinoma through induction of proMMP9 and its activation. Cell Microbiol. 2014;16(1):131-45.

38. Gao S, Li S, Ma Z, Liang S, Shan T, Zhang M, et al. Presence of Porphyromonas gingivalis in esophagus and its association with the clinicopathological characteristics and survival in patients with esophageal cancer. Infect Agent Cancer. 2016;11:3.

39. Gallimidi A, Fischman S, Revach B, Bulvik R, Maliutina A, Rubinstein AM, et al. Periodontal pathogens Porphyromonas gingivalis and Fusobacterium nucleatum promote tumor progression in an oral-specific chemical carcinogenesis model. Oncotarget. 2015;6(26):22613-23.

40. Ha NH, Woo BH, Kim DJ, Ha ES, Choi JI, Kim SJ, et al. Prolonged and repetitive exposure to Porphyromonas gingivalis increases aggressiveness of oral cancer cells by promoting acquisition of cancer stem cell properties. Tumour Biol. 2015;36(12):9947-60.

41. Nishimura F, Iwamoto Y, Mineshiba J, Shimizu A, Soga Y, Murayama Y. Periodontal disease and diabetes mellitus: the role of tumor necrosis factoralpha in a 2-way relationship. J Periodontol. 2003;74(1):97-102.

42. Yki JH, Sammalkorpi K, Koivisto VA, Nikkila EA. Severity, duration, and mechanisms of insulin resistance during acute infections. J Clin Endocrinol Metab. 1989;69(2):317-23.

43. Fernandez RJM, Lopez BA, Vendrell J, Ferri MJ, Recasens M, Ricart W. Burden of infection and insulin resistance in healthy middle-aged men. Diabetes Care. 2006;29(5):1058-64.

44. Teeuw WJ, Gerdes VE, Loos BG. Effect of periodontal treatment on glycemic control of diabetic patients: a systematic review and meta-analysis. Diabetes Care. 2010;33(2):421-7.

45. Mealey BL, Ocampo GL. Diabetes mellitus and periodontal disease. Periodontol 2000. 2007;44:12753. 
46. Xavier AC, Silva IN, Costa Fde O, Correa DS. Periodontal status in children and adolescents with type 1 diabetes mellitus. Arq Bras Endocrinol Metabol. 2009;53(3):348-54.

47. Kothari M, Spin NR, Nielsen JF. Comprehensive oral-health assessment of individuals with acquired brain-injury in neuro-rehabilitation setting. Brain Inj. 2016;30(9):1103-8.

48. Akiyama H, Barger S, Barnum S, Bradt B, Bauer J, Cole GM, et al. Inflammation and Alzheimer's disease. Neurobiol Aging. 2000;21(3):383-421.

49. Gaur S, Agnihotri R. Alzheimer's disease and chronic periodontitis: is there an association?. Geriatr Gerontol Int. 2015;15(4):391-404.
50. Bui FQ, Almeida SCLC, Huynh B, Trinh A, Liu J, Woodward J, et al. Association between periodontal pathogens and systemic disease. Biomed J. 2019;42(1):27-35.

Cite this article as: Albeshr AG, Alhabdan YA, Aljasem RM, Munshi MA, Bagasi AM, Khars MYA, et al. Gradings of periodontitis in relation with systemic disease. Int J Community Med Public Health 2021;8:3671-6. 